\title{
Efficacy of kissing balloon inflation after provisional stenting in bifurcation lesions guided by intravascular ultrasound: short and midterm results of the J-REVERSE registry
}

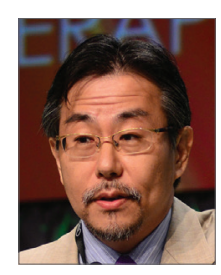

\author{
Yoshinobu Murasato ${ }^{1,2,13 *}, \mathrm{MD}, \mathrm{PhD}$; Yoshihisa Kinoshita ${ }^{3}, \mathrm{MD}$; \\ Masahiro Yamawaki ${ }^{4}, \mathrm{MD}, \mathrm{PhD}$; Toshiro Shinke ${ }^{5}, \mathrm{MD}, \mathrm{PhD}$; Hiromasa Otake $, \mathrm{MD}, \mathrm{PhD}$; \\ Yoshihiro Takeda ${ }^{6}, \mathrm{MD}, \mathrm{PhD}$; Kenichi Fujii ${ }^{7}, \mathrm{MD}, \mathrm{PhD}$; Shin-ichiro Yamada ${ }^{8}, \mathrm{MD}, \mathrm{PhD}$; \\ Yoshihisa Shimada ${ }^{9}$, MD, PhD, FAPSIC, FSCAI; Takehiro Yamashita ${ }^{10}$, MD, PhD, FACC; \\ Kazuhiko Yumoto ${ }^{11}$, MD, PhD; Yoshihisa Fujino ${ }^{12}, \mathrm{MD}, \mathrm{MPH}, \mathrm{PhD}$; \\ on behalf of the J-REVERSE Investigators
}

1. Department of Cardiology, Kyushu Medical Center, Fukuoka, Japan; 2. Clinical Research Institute, Kyushu Medical Center, Fukuoka, Japan; 3. Department of Cardiovascular Medicine, Toyohashi Heart Center, Toyohashi, Japan; 4. Department of Cardiovascular Medicine, Saiseikai Yokohama City Eastern Hospital, Yokohama, Japan; 5. Department of Cardiovascular Medicine, Kobe University, Kobe, Japan; 6. Department of Cardiology, Rinku General Medical Center, Izumi-Sano, Japan; 7. Department of Cardiovascular Medicine, Hyogo Medical University, Nishinomiya, Japan; 8. Department of Cardiovascular Medicine, Himeji Cardiovascular Center, Himeji, Japan; 9. Department of Cardiovascular Medicine, Cardiovascular Center, Shiroyama Hospital, Habikino, Japan; 10. Department of Cardiovascular Medicine, Cardiovascular Center Hokkaido Ono Hospital, Sapporo, Japan; 11. Department of Cardiovascular Medicine, Yokohama Rosai Hospital, Yokohama, Japan; 12. Department of Preventive Medicine and Community Health, University of Occupational and Environmental Health, Kitakyushu, Japan; 13. Department of Cardiovascular Medicine, New Yukuhashi Hospital, Yukuhashi, Japan

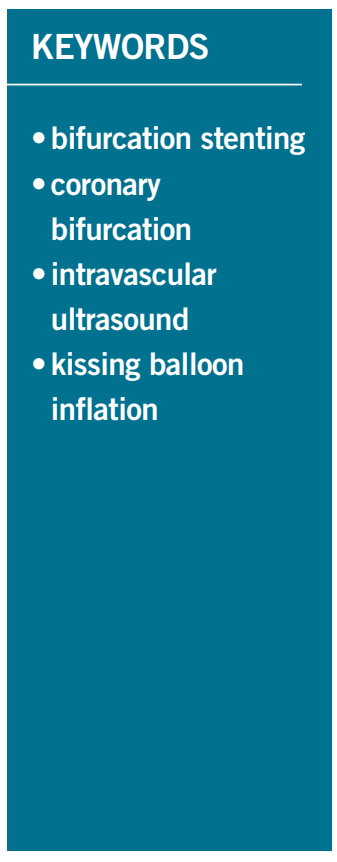

\begin{abstract}
Aims: Our aim was to investigate the efficacy of the kissing balloon technique (KBT) in the provisional stenting of bifurcation lesions guided by intravascular ultrasound.

Methods and results: In the J-REVERSE registry, 300 non-left main bifurcation lesions in 299 patients were divided into two groups, KBT $(n=163)$ and non-KBT $(n=137)$. Patient and lesion characteristics were similar except for a higher prevalence of diabetic patients in the non-KBT group. The major adverse cardiac events (MACE) incidence at nine-month follow-up, including target lesion revascularisation, myocardial infarction, stent thrombosis, and death, was $6.3 \%$ in the KBT group versus $9.1 \%$ in the non-KBT group $(\mathrm{p}=0.47)$. Regardless of more SB dissection $(10.5 \%$ vs. $1.5 \%, \mathrm{p}=0.001)$, the KBT group obtained a greater luminal volume in the proximal main vessel (MV) $\left(7.8 \pm 2.3\right.$ vs. $\left.7.0 \pm 2.0 \mathrm{~mm}^{3} / \mathrm{mm}, \mathrm{p}=0.006\right)$, maintained larger minimal lumen diameter at follow-up $(2.73 \pm 0.43$ vs. $2.63 \pm 0.39 \mathrm{~mm}, \mathrm{p}=0.04)$, and demonstrated less binary side branch $(\mathrm{SB})$ restenosis $(9.7 \%$ vs. $21.0 \%, \mathrm{p}=0.0004)$, which was beneficial for both true and non-true bifurcation lesions. Multivariate analysis showed the efficacy of KBT on SB restenosis (OR 0.28, 95\% CI: 0.12-0.62, $\mathrm{p}=0.002$ ).
\end{abstract}

Conclusions: Although the KBT increased SB dissection, it provided greater luminal gain in the proximal MV and SB with a similar MACE rate to non-KBT treatment up to nine-month follow-up.

*Corresponding author: Department of Cardiology, Cardiovascular Center, Kyushu Medical Center, 1-8-1, Jigyohama, Chuo, Fukuoka, 810-8563 Japan. E-mail: y.murasato@gmail.com 


\section{Introduction}

Provisional stenting of coronary bifurcation lesions is generally accepted as a first-line treatment ${ }^{1}$ because recent randomised studies and meta-analyses have demonstrated no greater decrease in adverse cardiac event rates after simultaneous deployment of stents in both the main vessel (MV) and a side branch $(\mathrm{SB})^{2-4}$. During provisional stenting, treatment with the kissing balloon technique (KBT), whereby MV and SB balloons are simultaneously inflated, has been considered effective after crossover MV stenting to secure SB patency, reduce SB stenosis, and remove jailed struts 5 . However, KBT efficacy after crossover stenting of bifurcation lesions has been questioned recently. In the NordicBaltic III trial, patients undergoing a single MV stent deployment and randomly assigned to final KBT or non-KBT demonstrated identical rates (2.9\%) of major adverse cardiac events (MACE) ${ }^{6}$. In the THUEBIS pilot trial, SB intervention was randomly assigned to mandatory versus provisional groups with similar MACE rates $(17.9 \%$ vs. $14.8 \%)$ despite a higher performance of KBT $(73.2 \%$ vs. $13.0 \%)^{7}$. In the Korean Coronary Bifurcation Stenting Registry (COBIS) trial, KBT was associated with a higher MACE rate than non-KBT $(8.7 \% \text { vs. } 4.7 \%)^{8}$.

Intravascular ultrasound (IVUS) optimises percutaneous coronary interventions (PCI), device selection, and lesion evaluation and has been reported to lower death, myocardial infarction (MI), target lesion revascularisation (TLR), and stent thrombosis rates after the deployment of a drug-eluting stent (DES) in bifurcation lesions ${ }^{9-11}$. However, in studies of bifurcation lesions including $>200$ patients, IVUS was actually performed in only $15-40 \%$ of patients ${ }^{2-4,6-8}$. While IVUS might be expected to confer benefits, the efficacy of KBT under complete IVUS guidance to treat bifurcation lesions has not been investigated.

This study, based on a prospective multicentre registry, compared the short and midterm results of IVUS-guided provisional stenting of coronary bifurcation lesions treated with and without KBT and investigated the efficacy of the KBT.

Editorial, see page 1209

\section{Study population and methods}

This study is described and registered as "J-REVERSE" (NCT01266239) on the US government registry of clinical trials (http://clinicaltrials.gov). The study complies with the guidelines of the Declaration of Helsinki and was reviewed and approved by the institutional review boards of all participating institutions.

A total of 303 de novo coronary bifurcation lesions in 302 patients, enrolled at 13 Japanese medical institutions, were treated with a XIENCE $V^{\circledR}$ everolimus-eluting stent (EES) (Abbott Vascular, Santa Clara, CA, USA) or CYPHER ${ }^{\circledR}$ Select Plus sirolimus-eluting stent (SES) (Cordis Corporation, Bridgewater, NJ, USA) between June 2010 and August 2012. The inclusion criteria were: 1) $\geq 75 \%$ MV stenosis with or without $\geq 75 \%$ SB stenosis, 2) $\geq 2.5 \mathrm{~mm} \mathrm{MV}$ and $\geq 2.0 \mathrm{~mm}$ SB reference diameters (RD) by visual estimates, 3) $\leq 46 \mathrm{~mm}$ lesion length, 4) lesion judged appropriate for provisional MV stenting, 5) mandatory pre- and post-procedural IVUS examination, and 6) age $\geq 20$ and $\leq 85$ years. Patients were excluded if they: 1) presented with a lesion involving the left main coronary bifurcation or trifurcation lesion, 2) were in the midst of acute MI and unstable angina with a thrombus-containing culprit vessel, 3) presented with $<30 \%$ left ventricular ejection fraction, 4) were in a state of circulatory shock, 5) were not candidates for dual antiplatelet therapy or intolerant of contrast material or radiation exposure, or 6 ) had a $<1$-year life expectancy. Three patients were excluded, and 300 lesions were ultimately retained for analysis.

\section{Percutaneous coronary intervention}

Crossover stenting was performed from the proximal to distal bifurcated MV in the lesion. The SB was dilated using KBT in 163 lesions (54\%) (Figure 1), either at the operator's discretion or when forced by a decrease in blood flow to <TIMI 3. Thirty-four and 129 of these lesions were treated with SES and EES in the MV, respectively. The SB was left jailed by the MV stent struts in the remaining 137 lesions (non-KBT group). Twenty-six and 111 of these lesions were treated with SES and EES, respectively. The $\mathrm{SB}$ was stented for $\leq \mathrm{TIMI} 1$ flow, major dissections, or development of symptoms consistent with myocardial ischaemia associated with electrocardiographic changes.

\section{Intravascular ultrasound}

IVUS examinations were performed before $\mathrm{PCI}$ in the MV and SB using Atlantis ${ }^{\circledR}$ SR Pro, Galaxy ${ }^{\circledR}$ or i-Lab ${ }^{\circledR}$ (Boston Scientific, Marlborough, MA, USA), Revolution ${ }^{\circledR}$ and Volcano $5^{\mathrm{TM}}$ (Volcano Corporation, San Diego, CA, USA), or ViewIT and Visiwave (Terumo, Tokyo, Japan) imaging systems. IVUS examination was performed in both arterial branches after KBT, and only in the MV in non-KBT cases. Automatic pullback at $0.5 \mathrm{~mm} / \mathrm{s}$ was performed after intracoronary injection of nitroglycerine from $>10 \mathrm{~mm}$ distal to the bifurcation lesion.

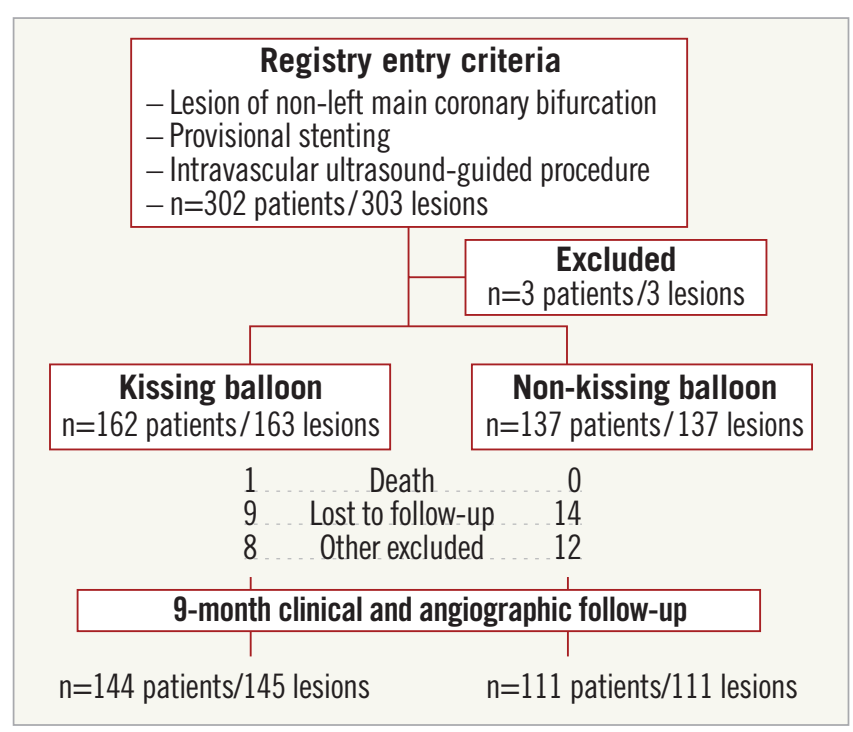

Figure 1. Flow of study groups from entry into the J-REVERSE registry to the end of the nine-month follow-up. 
Based on IVUS observations, the MV stent size and length were determined to match the lumen area of healthy distal MV and cover the entire atherosclerotic lesion. The SB balloon was also selected according to the distal reference lumen area. IVUS criteria for optimal bifurcation PCI were the following: (1) minimal stent area $\geq 55 \%$ of the average of proximal and distal MV reference crosssectional areas $(\mathrm{CSA})^{12}$ in the measurement on site, (2) no malapposition of the struts, and (3) no flow-limiting dissection or flap in either the edge of the stent or the SB. When malapposition of the stent was observed in the proximal MV, KBT was first performed with higher pressure, and proximal optimisation with a larger balloon was applied in the event of KBT failure in complete strut apposition. The SB stent was deployed only when the flap threatened total occlusion even after SB ballooning to optimal size.

\section{Periprocedural pharmaceutical regimens}

All patients received dual antiplatelet therapy with aspirin $81-200 \mathrm{mg}$ daily and clopidogrel $75 \mathrm{mg}$ or ticlopidine $200 \mathrm{mg}$ daily for $>1$ week before PCI, or a $300 \mathrm{mg}$ loading dose of clopidogrel on the day of PCI, which was continued for $\geq 9$ months. During PCI, intravenous, unfractionated heparin was administered in a 70-100 $\mathrm{U} / \mathrm{kg}$ bolus, with additional doses administered to maintain an activated clotting time of approximately $300 \mathrm{~s}$.

\section{Follow-up and study endpoints}

Nine months after PCI, regardless of clinical status, 274 patients (92\%) were examined, and $256(86 \%)$ underwent scheduled follow-up coronary angiography. Coronary angiography was performed ahead of schedule in four patients with symptoms consistent with myocardial ischaemia. The endpoints of the study were classified as: 1) procedure-related complications (including SB dissection, occlusion, or stenting; STIMI 1 flow across either bifurcation branch; and MI with increase in serum creatinine phosphokinase concentration to $\geq 3$ times the upper normal limit), and 2) MACE during the follow-up period (including cardiac death, MI, TLR, and stent thrombosis).

\section{Angiographic analysis}

Pre-PCI, post-PCI, and follow-up angiographic data were collected and analysed by an independent core laboratory (Cardiovascular Imaging Center, Toyohashi, Japan). Quantitative coronary angiography was performed using QAngio ${ }^{\circledR}$ XA 7.2 software (Medis medical imaging systems, Leiden, The Netherlands) with a dedicated analysis function validated for bifurcation lesions and $\mathrm{a} \geq 5 \mathrm{Fr}$ dye-filled catheter as RD. After excluding lesions with low image quality, we analysed 293 lesions pre and post PCI, and 256 lesions at follow-up coronary angiography. The $\mathrm{RD}$, minimum lumen diameter (MLD), and \% diameter stenosis (\%DS), defined as $100 \times(1-$ $[\mathrm{MLD} / \mathrm{RD}])$, were measured in the proximal and distal MV and SB.

\section{Intravascular ultrasound analysis}

Pre- and post-PCI IVUS data were also analysed by the independent core laboratory. After excluding lesions with low image quality,
250 lesions were retained for analysis. The bifurcation was divided into three segments: 1) proximal MV $(6 \mathrm{~mm})$ originating from the $\mathrm{SB}$ ostium, 2) distal MV (6 mm), and 3) SB (5 mm) originating from the tip of the carina in the direction of each branch (Figure 2A). In total, we quantitatively analysed 5,250 segments in $1 \mathrm{~mm}$ slices on all bifurcation branches using EchoPlaque planimetry software (INDEC Medical Systems, Santa Clara, CA, USA). The lumen and external elastic membrane (EEM) contours were imaged in the reference and minimal lumen segments, and volumetric analysis was performed using Simpson's rule. The EEM and lumen volumetric indexes were calculated as their respective volumes divided by the measured length. The eccentricity index was defined as the ratio of maximal to minimal diameter of the cross-section.

A
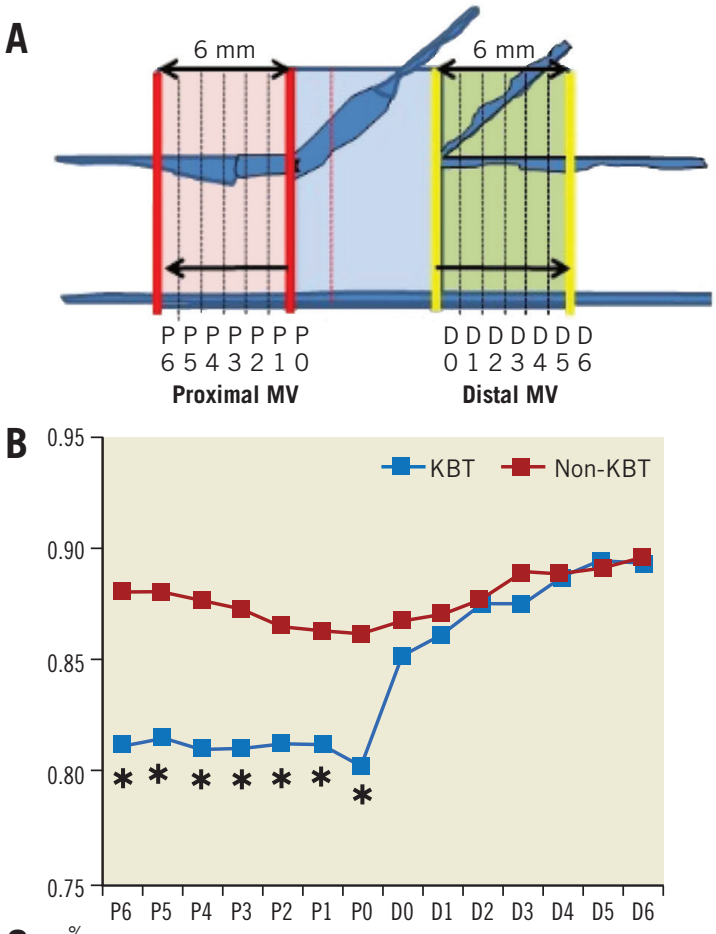

C

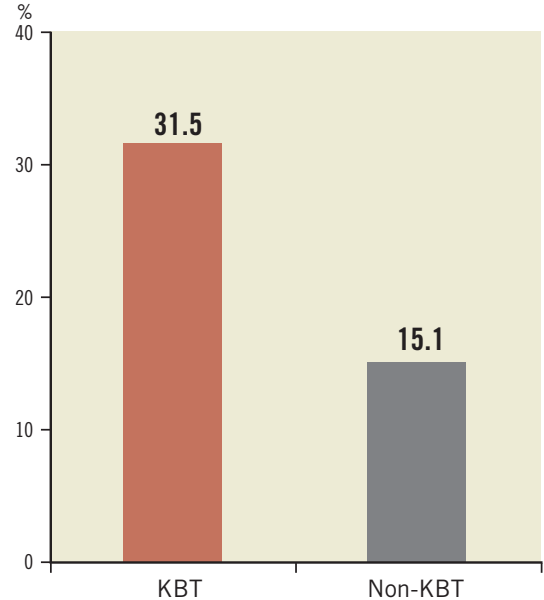

Figure 2. IVUS analysis. A) Analysed area in the main vessel (MV). $B)$ Stent eccentricity index in the $M V$. $* B T$ vs. Non-KBT group, $p<0.05$. C) Percentage of lumen volume increment of the proximal compared to the distal MV. KBT vs. Non-KBT, $p=0.000001$. 


\section{Statistical analysis}

The data are expressed as mean $\pm \mathrm{SD}$ or counts and percentages. Mean individual values were compared using the Student's paired t-test, while between-group comparisons were examined using the Student's unpaired t-test. Between-group differences in counts and percentages were examined using the chi-square test, corrected by Fisher's exact test where appropriate. Univariate and multivariate odds ratios (OR) of KBT for MACE and SB restenosis were estimated by the logistic regression model. The multivariate model included KBT, age, hypertension, diabetes mellitus, dyslipidaemia, smoking history, acute coronary syndrome or unstable angina, three-vessel disease, true bifurcation lesion, bifurcation angle $>70$ degrees, stent size, stent length, inflation pressure, MLD and $\% \mathrm{DS}$ of each branch pre and post PCI as possible confounding factors. All p-values were two-sided and considered statistically significant at $<0.05$. All statistical analyses were performed with EZR software (Saitama Medical Center, Jichi Medical University, Saitama, Japan), a modified version of $\mathrm{R}$ Commander (The $\mathrm{R}$ Foundation for Statistical Computing, Vienna, Austria) $)^{13}$.

\section{Results}

\section{BASELINE CLINICAL AND ANGIOGRAPHIC CHARACTERISTICS}

Stable angina and silent ischaemia were present in $>85 \%$ of patients in both groups. Baseline patient characteristics were similar between groups except for a lower prevalence of diabetes mellitus (39\% vs. 51\%) and insulin users (3\% vs. 10\%) in the KBT group (Table 1). Lesion characteristics including distribution, Medina classification, and true bifurcation were also similar (Table 2).

\section{PROCEDURAL OBSERVATIONS}

Procedural characteristics are shown in Table 3. In both groups, approximately $80 \%$ of patients were treated from the radial artery with 6 Fr guiding catheters, and another stent was deployed proximally or distally to the first in approximately $20 \%$. Size, length, and delivery pressure of the stents were similar (Table 3). MV and SB predilation was performed at similar rates, approximately $70 \%$ and $17 \%$, respectively, using balloons of similar size and length.

\section{INTRAVASCULAR ULTRASOUND ANALYSIS}

The IVUS success criterion of minimal stent area $\geq 55 \%$ of the average of the proximal and distal MV reference CSAs was achieved in $97 \%$ of proximal MV and $91 \%$ of distal MV. The proximal MV eccentricity index was similar to that of the distal MV (0.86-0.88) in the non-KBT group and significantly reduced to $0.80-0.82$ with KBT (Figure 2B). Quantitative two-dimensional and volumetric IVUS analyses revealed no significant between-group differences in pre-PCI luminal, EEM, or plaque CSAs or volumetric analysis in each branch (Table 4). Significantly greater proximal MV lumen enlargement was obtained in the KBT group compared to the non-KBT group in two-dimensional (CSA: 6.8 \pm 2.1 vs. $\left.6.1 \pm 1.7 \mathrm{~mm}^{2}, \mathrm{p}=0.002\right)$ and volumetric analyses $(7.8 \pm 2.3$ vs.
Table 1. Characteristics of the study groups.

\begin{tabular}{|c|c|c|c|}
\hline & $\begin{array}{c}\text { Kissing } \\
\text { balloon, } \\
\text { n=162 } \\
\text { patients }\end{array}$ & $\begin{array}{c}\text { Non-kissing } \\
\text { balloon, } \\
\text { n=137 } \\
\text { patients }\end{array}$ & $p$-value \\
\hline Age (years) & $67.6 \pm 8.5$ & $67.6 \pm 10.0$ & 0.95 \\
\hline Male sex, n (\%) & $131(80.9)$ & $111(81.0)$ & 1.00 \\
\hline Body mass index $\left(\mathrm{kg} / \mathrm{m}^{2}\right)$ & $23.6 \pm 3.3$ & $24.1 \pm 3.1$ & 0.21 \\
\hline \multicolumn{4}{|l|}{ Coronary risk factors } \\
\hline Hypertension, n (\%) & $120(74.1)$ & $110(80.2)$ & 0.22 \\
\hline Dyslipidaemia, n (\%) & $118(72.8)$ & $101(73.7)$ & 0.17 \\
\hline $\mathrm{LDL}(\mathrm{mg} / \mathrm{dL})$ & $110.1 \pm 31.8$ & $107.9 \pm 31.3$ & 0.63 \\
\hline Statin treatment, n (\%) & $95(58.6)$ & $83(60.6)$ & 0.72 \\
\hline Diabetes mellitus, $\mathrm{n}(\%)$ & $63(38.9)$ & $70(51.1)$ & 0.04 \\
\hline $\mathrm{HbA1C}(\%)$ & $5.9 \pm 0.9$ & $6.2 \pm 1.4$ & 0.03 \\
\hline Insulin user, n (\%) & $5(3.1)$ & $14(10.3)$ & 0.02 \\
\hline Current smoker, n (\%) & $48(29.6)$ & $35(25.5)$ & 0.52 \\
\hline Haemodialysis, n (\%) & $6(3.7)$ & $7(5.1)$ & 0.58 \\
\hline \multicolumn{4}{|l|}{ Medical history } \\
\hline $\begin{array}{l}\text { Prior myocardial } \\
\text { infarction, } \mathrm{n}(\%)\end{array}$ & $24(14.8)$ & $27(19.7)$ & 0.28 \\
\hline Prior heart failure, $\mathrm{n}(\%)$ & $10(6.2)$ & $11(8.0)$ & 0.65 \\
\hline Prior CABG, n (\%) & $2(1.2)$ & $1(0.7)$ & 1.00 \\
\hline $\begin{array}{l}\text { Prior coronary } \\
\text { intervention, } \mathrm{n}(\%)\end{array}$ & $59(36.4)$ & $48(14.3)$ & 0.90 \\
\hline \multicolumn{4}{|l|}{ Clinical presentation } \\
\hline Unstable angina, n (\%) & $29(17.9)$ & $18(13.1)$ & 0.37 \\
\hline Stable angina, $\mathrm{n}(\%)$ & $116(71.6)$ & $99(72.3)$ & \\
\hline $\begin{array}{l}\text { Silent myocardial } \\
\text { ischaemia, } \mathrm{n}(\%)\end{array}$ & $17(10.5)$ & $20(14.3)$ & \\
\hline $\begin{array}{l}\text { Left ventricular ejection } \\
\text { fraction }(\%)\end{array}$ & $62 \pm 13$ & $60 \pm 15$ & 0.13 \\
\hline \multicolumn{4}{|c|}{$\begin{array}{l}\text { Values are mean } \pm S D \text { or number }(\%) \text { of observations. CABG: coronary } \\
\text { artery bypass graft }\end{array}$} \\
\hline
\end{tabular}

$\left.7.0 \pm 2.0 \mathrm{~mm}^{3} / \mathrm{mm}, \mathrm{p}=0.006\right)$. The percentage of lumen volume increment of the proximal compared to the distal MV was significantly larger in the KBT group $(31.5 \pm 26.7 \%$ vs. $15.1 \pm 25.4 \%$, $\mathrm{p}=0.000001$ ) (Figure 2C). Pre-PCI SB parameters did not differ significantly between the two groups. KBT was associated with significant increases in EEM volume $\left(6.8 \pm 2.2\right.$ vs. $6.2 \pm 2.2 \mathrm{~mm}^{3} /$ $\mathrm{mm}, \mathrm{p}=0.000004)$, lumen volume $\left(3.6 \pm 2.0\right.$ vs. $3.2 \pm 1.3 \mathrm{~mm}^{3} / \mathrm{mm}$, $\mathrm{p}=0.00002)$, and plaque volume $\left(3.1 \pm 2.0\right.$ vs. $3.0 \pm 1.4 \mathrm{~mm}^{3} / \mathrm{mm}$, $\mathrm{p}=0.004$ ) compared to pre-PCI. At the minimal lumen area (MLA) site, KBT also increased EEM ( $6.5 \pm 1.9$ vs. $\left.6.0 \pm 2.0 \mathrm{~mm}^{2}, \mathrm{p}=0.0002\right)$ and lumen CSAs $\left(2.8 \pm 1.0\right.$ vs. $\left.2.4 \pm 1.1 \mathrm{~mm}^{2}, \mathrm{p}=0.0000002\right)$, while plaque area percentage decreased $(55.6 \pm 12.9 \%$ vs. $57.9 \pm 15.4 \%$, $\mathrm{p}=0.014$ ) due to the absence of significant change in plaque CSA $(\mathrm{p}=0.92)$.

\section{PROCEDURE-RELATED COMPLICATIONS}

Although more frequent flow-limiting SB dissections $(10.5 \%$ vs. $1.5 \%, \mathrm{p}=0.001)$ and stenting $(5.6 \%$ vs. $0.7 \%, \mathrm{p}=0.024)$ were 
Table 2. Lesion distribution and characteristics.

\begin{tabular}{|c|c|c|c|}
\hline & \begin{tabular}{|c|} 
Kissing \\
balloon, \\
$\mathrm{n}=163$ lesions
\end{tabular} & $\begin{array}{c}\text { Non-kissing } \\
\text { balloon, } \\
n=137 \text { lesions }\end{array}$ & $p$-value \\
\hline \multicolumn{4}{|c|}{ Number of diseased vessels, $n(\%)$} \\
\hline 1 & $91(55.8)$ & $72(52.5)$ & 0.07 \\
\hline 2 & $54(33.1)$ & $37(27.0)$ & \\
\hline 3 & $18(11.0)$ & $28(20.4)$ & \\
\hline \multicolumn{4}{|c|}{ Culprit coronary artery, n (\%) } \\
\hline Left anterior descending & $124(76.1)$ & $91(66.4)$ & 0.17 \\
\hline Left circumflex & $23(14.1)$ & $29(21.2)$ & \\
\hline Right & $16(9.8)$ & $17(12.4)$ & \\
\hline \multicolumn{4}{|c|}{ Medina classification, $\mathrm{n}(\%)$} \\
\hline $1,1,1$ & $56(34.4)$ & $37(27.0)$ & 0.17 \\
\hline $1,0,1$ & $10(6.1)$ & $5(3.6)$ & \\
\hline $0,1,1$ & $21(12.9)$ & $20(14.6)$ & \\
\hline $1,1,0$ & $28(17.2)$ & $32(23.4)$ & \\
\hline $1,0,0$ & $13(8.0)$ & $20(14.6)$ & \\
\hline $0,1,0$ & $30(18.4)$ & $22(16.1)$ & \\
\hline $0,0,1$ & $4(2.5)$ & $0(0)$ & \\
\hline True bifurcation lesion & $87(53.4)$ & $62(45.2)$ & 0.17 \\
\hline
\end{tabular}

observed in the KBT group, SB occlusion, MI, and slow/no-flow rates were similar between groups (Figure 3 ). The KBT group had a higher overall incidence of procedure-related complications $(12.9 \%$ vs. $5.8 \%, \mathrm{p}=0.049)$.

\section{ANGIOGRAPHIC OBSERVATIONS}

Pre-PCI MLD and \%DS of the proximal and distal MV were similar between groups, while greater stenosis was observed regardless of larger RD in the SB of the KBT group (Table 5). Post PCI, we observed trends for larger MLD and shorter lesion length in the proximal MV and significantly larger MLD $(1.47 \pm 0.43 \mathrm{~mm}$ vs. $1.23 \pm 0.50 \mathrm{~mm}, \mathrm{p}=0.00002)$ and lower $\% \mathrm{DS}(33.5 \pm 16.5 \%$ vs. $41.6 \pm 20.5 \%, \mathrm{p}=0.0002)$ in the $\mathrm{SB}$ in the KBT group. At ninemonth follow-up, significantly larger MLDs in the proximal MV

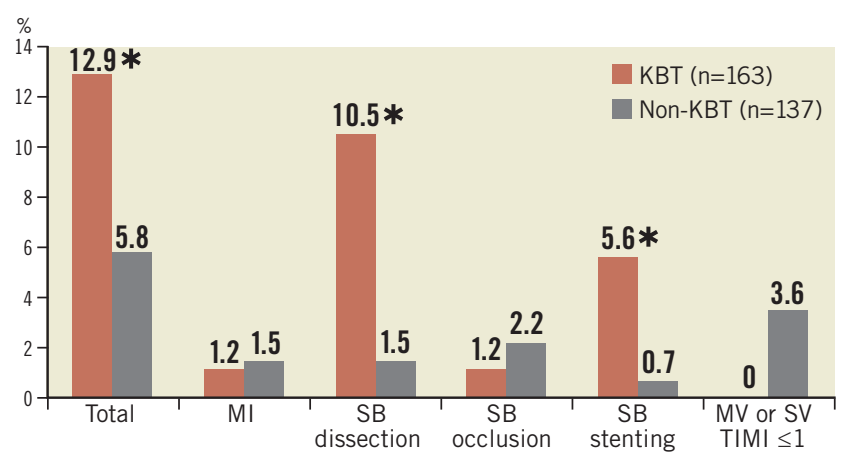

Figure 3. Procedure-related complications in the acute phase in the KBT versus non-KBT groups. * KBT vs. Non-KBT group, $p<0.05$
Table 3. Procedural characteristics in whole cohort.

\begin{tabular}{|l|c|c|c|}
\hline & $\begin{array}{c}\text { Kissing } \\
\text { balloon, } \\
\text { n=163 lesions }\end{array}$ & $\begin{array}{c}\text { Non-kissing } \\
\text { balloon, } \\
\text { n=137 lesions }\end{array}$ & p-value \\
\hline Approach, n (\%) & $35(21.5)$ & $30(21.9)$ & 1.00 \\
\hline Femoral & $128(78.5)$ & $107(78.1)$ & \\
\hline Radial & $132(81.0)$ & $109(79.6)$ & 0.94 \\
\hline Guiding catheter, n (\%) & $26(16.0)$ & $24(17.5)$ & \\
\hline $6 \mathrm{Fr}$ & $5(3.1)$ & $4(2.9)$ & \\
\hline $7 \mathrm{Fr}$ & &
\end{tabular}

Predilation, $\mathbf{n}(\%)$

\begin{tabular}{|l|r|r|r|}
\hline Main vessel & $116(71.2)$ & $94(68.6)$ & 0.71 \\
\hline Side branch & $29(17.8)$ & $22(16.0)$ & 0.76 \\
\hline
\end{tabular}

Additional main vessel stenting, $\mathrm{n}(\%)$

\begin{tabular}{|l|l|l|l|}
\hline Proximal & $20(12.3)$ & $13(9.5)$ & 0.47 \\
\hline Distal & $17(10.4)$ & $13(9.5)$ & 0.85 \\
\hline
\end{tabular}

Main vessel stent

\begin{tabular}{|l|c|c|c|}
\hline Diameter, $\mathrm{mm}$ & $2.97 \pm 0.34$ & $2.95 \pm 0.34$ & 0.74 \\
\hline Length, $\mathrm{mm}$ & $22.2 \pm 5.2$ & $21.1 \pm 5.4$ & 0.07 \\
\hline Pressure, $\mathrm{mmHg}$ & $11.1 \pm 2.8$ & $11.4 \pm 3.0$ & 0.27
\end{tabular}

\section{Additional main vessel stent}

\begin{tabular}{|l|c|c|c|}
\hline Diameter, $\mathrm{mm}$ & $3.07 \pm 0.37$ & $2.90 \pm 0.30$ & 0.07 \\
\hline Length, $\mathrm{mm}$ & $18.6 \pm 6.8$ & $18.3 \pm 4.9$ & 0.87 \\
\hline Pressure, $\mathrm{mmHg}$ & $12.2 \pm 2.7$ & $11.3 \pm 2.7$ & 0.20
\end{tabular}

\section{Predilation balloon}

Main vessel

\begin{tabular}{|c|c|c|c|}
\hline Diameter, $\mathrm{mm}$ & $2.64 \pm 0.42$ & $2.62 \pm 0.40$ & 0.77 \\
\hline Length, $\mathrm{mm}$ & $14.5 \pm 3.0$ & $14.0 \pm 3.3$ & 0.30 \\
\hline
\end{tabular}

Side branch

\begin{tabular}{|c|c|c|c|}
\hline Diameter, $\mathrm{mm}$ & $2.11 \pm 0.28$ & $2.26 \pm 0.45$ & 0.15 \\
\hline Length, $\mathrm{mm}$ & $13.8 \pm 2.0$ & $14.0 \pm 3.2$ & 0.69 \\
\hline
\end{tabular}

\section{Kissing balloon inflation}

\begin{tabular}{|c|c|c|c|}
\hline \multicolumn{4}{|l|}{ Main vessel } \\
\hline Diameter, mm & $2.93 \pm 0.41$ & ND & $(-)$ \\
\hline Length, mm & $14.3 \pm 3.9$ & ND & $(-)$ \\
\hline Pressure, $\mathrm{mmHg}$ & $9.6 \pm 3.6$ & ND & $(-)$ \\
\hline \multicolumn{4}{|l|}{ Side branch } \\
\hline Diameter, mm & $2.20 \pm 0.28$ & ND & $(-)$ \\
\hline Length, mm & $14.2 \pm 2.5$ & ND & $(-)$ \\
\hline Pressure, $\mathrm{mmHg}$ & $9.0 \pm 2.9$ & ND & $(-)$ \\
\hline
\end{tabular}

Values are mean \pm SD or number (\%) of observations. ND: not detectable

(2.73 $\pm 0.43 \mathrm{~mm}$ vs. $2.63 \pm 0.3 \mathrm{~mm}, \mathrm{p}=0.04)$ and $\mathrm{SB}(1.54 \pm 0.44 \mathrm{~mm}$ vs. $1.21 \pm 0.51 \mathrm{~mm}, \mathrm{p}=0.0000001)$ were maintained in the KBT group. The $\% \mathrm{DS}$ in the SB was further reduced compared to postPCI values in the KBT group, whereas it worsened post PCI and did not improve at follow-up in the non-KBT group (Figure 4A, Figure 4B). Similar late loss rates were observed in the proximal and distal MV (Figure 4C). 
Table 4. Two-dimensional and volumetric intravascular ultrasound analysis.

\begin{tabular}{|c|c|c|c|c|c|c|c|c|c|}
\hline & \multicolumn{3}{|c|}{ Proximal main vessel } & \multicolumn{3}{|c|}{ Distal main vessel } & \multicolumn{3}{|c|}{ Side branch } \\
\hline & $\begin{array}{l}\text { Kissing } \\
\text { balloon }\end{array}$ & $\begin{array}{c}\text { Non-kissing } \\
\text { balloon }\end{array}$ & $p$-value & $\begin{array}{l}\text { Kissing } \\
\text { balloon }\end{array}$ & $\begin{array}{l}\text { Non-kissing } \\
\text { balloon }\end{array}$ & $p$-value & $\begin{array}{l}\text { Kissing } \\
\text { balloon }\end{array}$ & $\begin{array}{c}\text { Non-kissing } \\
\text { balloon }\end{array}$ & $p$-value \\
\hline \multicolumn{10}{|c|}{ Two-dimensional analysis } \\
\hline \multicolumn{10}{|l|}{ Reference } \\
\hline lumen CSA (mm²) & $9.0 \pm 2.9$ & $9.2 \pm 2.6$ & 0.58 & $5.4 \pm 2.1$ & $5.8 \pm 2.4$ & 0.19 & $4.2 \pm 1.5$ & $4.2 \pm 1.4$ & 0.97 \\
\hline EEM CSA $\left(\mathrm{mm}^{2}\right)$ & $15.7 \pm 4.6$ & $16.6 \pm 4.5$ & 0.14 & $8.3 \pm 3.2$ & $9.0 \pm 3.5$ & 0.13 & $6.4 \pm 2.1$ & $6.7 \pm 2.2$ & 0.37 \\
\hline plaque CSA $\left(\mathrm{mm}^{2}\right)$ & $6.8 \pm 2.9$ & $7.5 \pm 2.8$ & 0.07 & $2.9 \pm 1.6$ & $3.2 \pm 1.6$ & 0.16 & $2.2 \pm 1.6$ & $2.5 \pm 1.6$ & 0.13 \\
\hline$\%$ plaque area & $41.9 \pm 11.4$ & $44.4 \pm 8.7$ & 0.06 & $33.3 \pm 10.2$ & $34.3 \pm 9.3$ & 0.43 & $33.4 \pm 11.1$ & $36.4 \pm 13.0$ & 0.15 \\
\hline \multicolumn{10}{|l|}{ Pre-MLA site } \\
\hline lumen CSA $\left(\mathrm{mm}^{2}\right)$ & $3.1 \pm 1.8$ & $3.1 \pm 1.8$ & 0.96 & $2.2 \pm 1.0$ & $2.4 \pm 1.4$ & 0.30 & $2.4 \pm 1.1$ & $2.5 \pm 1.1$ & 0.65 \\
\hline EEM CSA $\left(\mathrm{mm}^{2}\right)$ & $13.3 \pm 4.0$ & $13.4 \pm 3.9$ & 0.78 & $9.1 \pm 3.2$ & $9.8 \pm 3.6$ & 0.12 & $6.0 \pm 2.0$ & $6.5 \pm 2.5$ & 0.15 \\
\hline plaque CSA $\left(\mathrm{mm}^{2}\right)$ & $10.1 \pm 3.6$ & $10.3 \pm 3.3$ & 0.73 & $6.8 \pm 3.1$ & $7.4 \pm 3.4$ & 0.19 & $3.7 \pm 1.9$ & $4.0 \pm 2.4$ & 0.32 \\
\hline$\%$ plaque area & $76.0 \pm 11.3$ & $76.9 \pm 10.6$ & 0.55 & $73.3 \pm 12.3$ & $73.9 \pm 12.8$ & 0.73 & $57.9 \pm 15.4$ & $58.4 \pm 18.4$ & 0.88 \\
\hline \multicolumn{10}{|l|}{ Post-MLA site } \\
\hline lumen CSA $\left(\mathrm{mm}^{2}\right)$ & $6.8 \pm 2.1$ & $6.1 \pm 1.7$ & 0.002 & $5.5 \pm 2.2$ & $5.5 \pm 1.8$ & 0.86 & $2.8 \pm 1.0$ & ND & $(-)$ \\
\hline EEM CSA $\left(\mathrm{mm}^{2}\right)$ & $15.6 \pm 4.2$ & $15.4 \pm 4.0$ & 0.64 & $11.9 \pm 4.5$ & $12.2 \pm 3.7$ & 0.58 & 6.51 .9 & ND & $(-)$ \\
\hline plaque CSA $\left(\mathrm{mm}^{2}\right)$ & $8.8 \pm 2.8$ & $9.3 \pm 3.0$ & 0.22 & $6.3 \pm 2.5$ & $6.6 \pm 2.6$ & 0.28 & $3.7 \pm 1.6$ & ND & $(-)$ \\
\hline$\%$ plaque area & $55.7 \pm 8.9$ & $59.9 \pm 8.2$ & 0.0002 & $53.1 \pm 8.9$ & $53.6 \pm 9.6$ & 0.69 & $55.6 \pm 12.9$ & ND & $(-)$ \\
\hline \multicolumn{10}{|l|}{ Volumetric analysis } \\
\hline \multicolumn{10}{|l|}{ Pre PCl } \\
\hline lumen $\left(\mathrm{mm}^{3} / \mathrm{mm}\right)$ & $5.0 \pm 2.6$ & $4.8 \pm 2.3$ & 0.74 & $3.3 \pm 1.4$ & $3.7 \pm 2.0$ & 0.06 & $3.2 \pm 1.3$ & $3.2 \pm 1.3$ & 0.29 \\
\hline $\operatorname{EEM}\left(\mathrm{mm}^{3} / \mathrm{mm}\right)$ & $13.8 \pm 4.3$ & $14.2 \pm 3.3$ & 0.63 & $9.3 \pm 3.2$ & $10.1 \pm 3.8$ & 0.07 & $6.2 \pm 2.2$ & $6.5 \pm 2.5$ & 0.45 \\
\hline plaque $\left(\mathrm{mm}^{3} / \mathrm{mm}\right)$ & $8.8 \pm 3.3$ & $9.3 \pm 3.2$ & 0.34 & $5.9 \pm 3.4$ & $6.3 \pm 3.1$ & 0.23 & $3.0 \pm 1.4$ & $3.2 \pm 2.2$ & 0.87 \\
\hline \multicolumn{10}{|l|}{ Post PCl } \\
\hline Iumen $\left(\mathrm{mm}^{3} / \mathrm{mm}\right)$ & $7.8 \pm 2.3$ & $7.0 \pm 2.0$ & 0.006 & $6.0 \pm 1.7$ & $6.3 \pm 1.9$ & 0.31 & $3.6 \pm 2.0$ & ND & $(-)$ \\
\hline $\operatorname{EEM}\left(\mathrm{mm}^{3} / \mathrm{mm}\right)$ & $16.1 \pm 4.1$ & $15.8 \pm 4.0$ & 0.65 & $11.6 \pm 3.7$ & $12.2 \pm 3.7$ & 0.20 & $6.8 \pm 2.2$ & ND & $(-)$ \\
\hline plaque $\left(\mathrm{mm}^{3} / \mathrm{mm}\right)$ & $8.3 \pm 2.6$ & $8.8 \pm 2.9$ & 0.12 & $5.6 \pm 2.3$ & $5.7 \pm 5.9$ & 0.27 & $3.1 \pm 2.0$ & ND & $(-)$ \\
\hline stent $\left(\mathrm{mm}^{3} / \mathrm{mm}\right)$ & $6.9 \pm 2.3$ & $6.2 \pm 2.1$ & 0.009 & $5.7 \pm 2.0$ & $5.6 \pm 2.0$ & 0.77 & ND & ND & $(-)$ \\
\hline
\end{tabular}
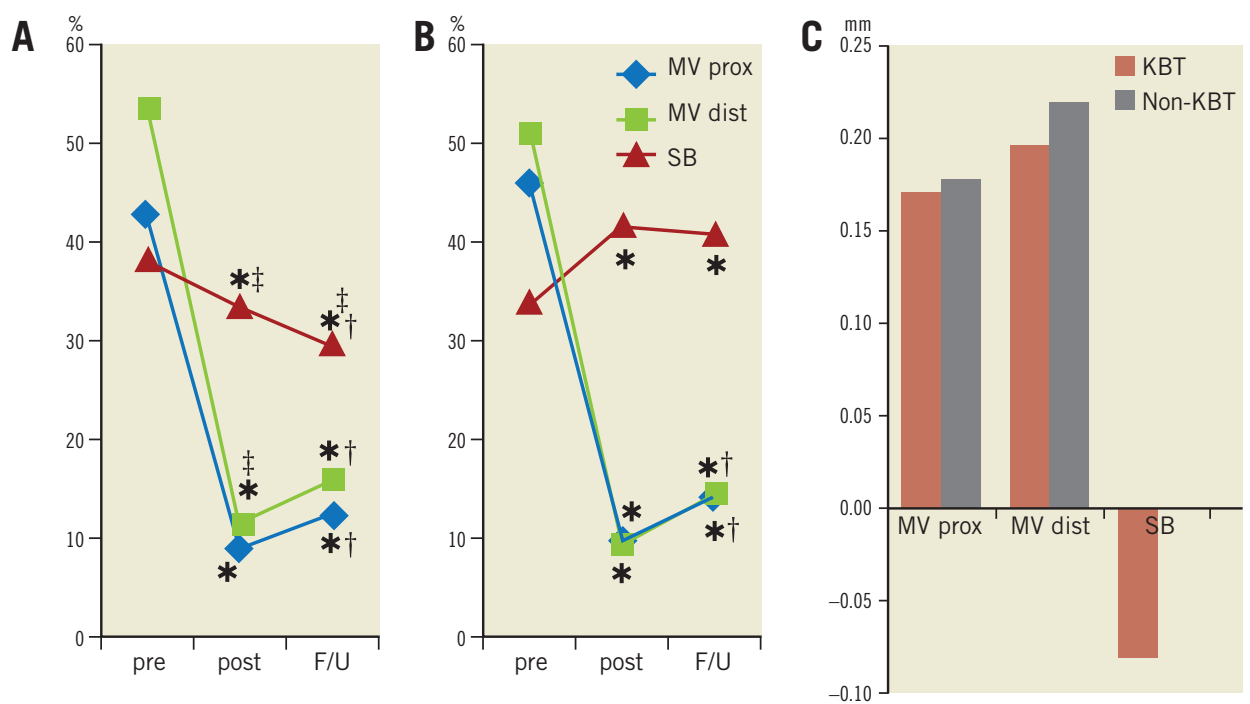

Figure 4. Percentage of diameter stenosis on quantitative coronary angiography analysis. A) KBT group. B) Non-KBT group. C) Lumen loss at nine-month follow-up. F/U: follow-up; MV prox: proximal main vessel; $M V$ dist: distal main vessel; pre: pre-PCI; post: post-PCI; SB: side branch; $*$ post-PCI or F/U vs. pre-PCI, $p<0.05 ; \uparrow F / U$ vs. post-PCI, $p<0.05 ; \neq$ KBT vs. Non-KBT group, $p<0.05$ 
Table 5. Pre- and post-procedural and nine-month quantitative coronary angiography.

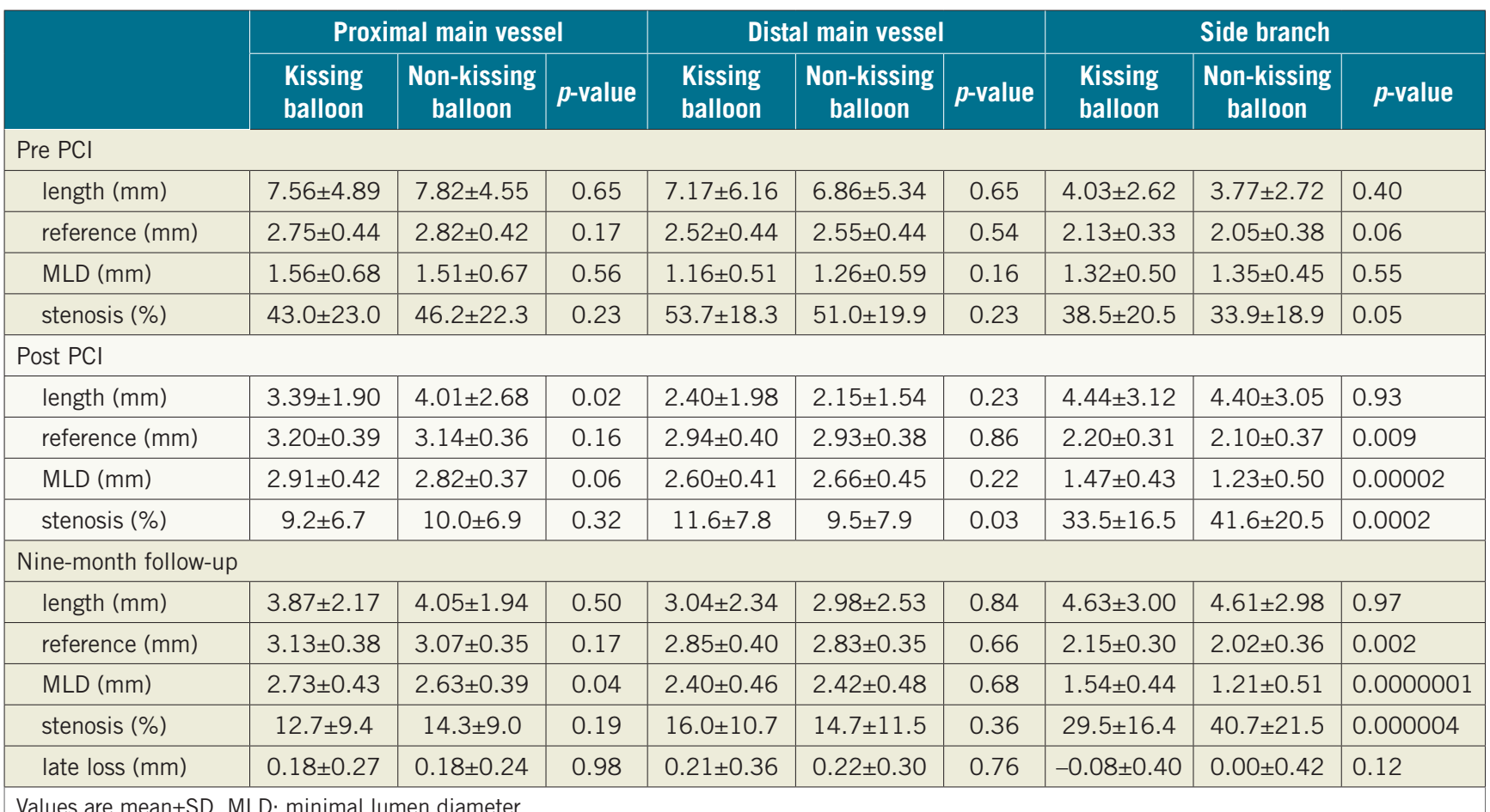

\section{CLINICAL OUTCOMES}

The cumulative nine-month MACE rate was $6.3 \%$ in the KBT versus $9.1 \%$ in the non-KBT group $(\mathrm{p}=0.47$ ) (Table 6). In the KBT group, one patient died from heart failure, and one patient

Table 6. Cumulative nine-month incidence of adverse clinical events in the kissing versus non-kissing balloon groups.

\begin{tabular}{|c|c|c|c|}
\hline & $\begin{array}{l}\text { Kissing } \\
\text { balloon } \\
(n=145)\end{array}$ & $\begin{array}{c}\text { Non-kissing } \\
\text { balloon } \\
(n=111)\end{array}$ & $p$-value \\
\hline $\begin{array}{l}\text { Major adverse cardiac events, } \\
\text { n (\%) }\end{array}$ & $9(6.3)$ & $10(9.1)$ & 0.47 \\
\hline Myocardial infarction, n (\%) & $1(0.7)$ & $2(1.8)$ & 0.58 \\
\hline Cardiac death, n (\%) & $1(0.7)$ & $0(0)$ & 1.00 \\
\hline $\begin{array}{l}\text { Definite/probable stent } \\
\text { thrombosis, n (\%) }\end{array}$ & $0(0)$ & $1(0.9)$ & 0.43 \\
\hline $\begin{array}{l}\text { Target lesion revascularisation, } \\
\mathrm{n}(\%)\end{array}$ & $8(5.5)$ & $9(8.1)$ & 0.45 \\
\hline \multicolumn{4}{|l|}{ Main vessel } \\
\hline In-stent, n (\%) & $1(0.7)$ & $7(6.3)$ & 0.02 \\
\hline Stent edge, n (\%) & $6(4.1)$ & $1(0.9)$ & 0.14 \\
\hline Side branch, n (\%) & $1(0.7)$ & $1(0.9)$ & 1.00 \\
\hline $\begin{array}{l}\text { Binary restenosis in the } \\
\text { bifurcation, } \mathrm{n}(\%)\end{array}$ & $16(11.0)$ & $33(29.7)$ & 0.0002 \\
\hline Proximal main vessel, $\mathrm{n}(\%)$ & $1(0.7)$ & $1(0.9)$ & 1.00 \\
\hline Distal main vessel, $\mathrm{n}(\%)$ & $1(0.7)$ & $2(1.8)$ & 0.58 \\
\hline Side branch, n (\%) & $14(9.7)$ & $30(27.0)$ & 0.0004 \\
\hline
\end{tabular}

suffered an MI. In the non-KBT group, two patients suffered an MI, one due to stent thrombosis. TLR was performed for eight lesions $(5.5 \%)$ in the KBT group compared with nine $(8.1 \%)$ in the non-KBT group. The TLR site was more likely to be inside the MV stent in the non-KBT group (6.3\% vs. $0.7 \%, \mathrm{p}=0.02)$. Binary restenosis with $>50 \%$ stenosis and worsening compared to postPCI values in the bifurcated area was also assessed. The bifurcated area was defined as the integrated area of each segment from the carina to sites $5 \mathrm{~mm}$ from it. Although the frequency of restenosis in the proximal and distal MV was very low in both groups, SB restenosis in the non-KBT group was significantly more frequent $(27.0 \%$ vs. $9.7 \%, \mathrm{p}=0.0004)$. In the logistic regression analysis, the KBT was not associated with MACE in either univariate or multivariate analysis (Table 7). On the contrary, the beneficial effect on SB restenosis was demonstrated in both univariate (OR $0.32,95 \% \mathrm{CI}: 0.16-0.62, \mathrm{p}=0.001)$ and multivariate analysis (OR 0.28, 95\% CI: 0.12-0.62, $\mathrm{p}=0.002$ ) (Table 8).

Lesions were divided into true $(\mathrm{n}=153)$ and non-true bifurcation lesions $(n=147)$ by visual estimation of Medina classification. In total, 91 true and 72 non-true bifurcation lesions were treated with KBT. In the true bifurcation lesion subgroup, MACE at ninemonth follow-up was non-significantly higher in lesions treated with non-KBT compared to KBT $(10.2 \%$ vs. $6.0 \%, \mathrm{p}=0.50)$, as was the TLR rate $(10.2 \%$ vs. $4.8 \%, \mathrm{p}=0.29)$. In the non-true bifurcation lesion subgroup, lesions treated with and without KBT did not differ significantly (MACE: $6.5 \%$ vs. $8.2 \%$ ). Lesions treated with and without KBT did not differ significantly in terms of the rates of proximal or distal MV binary restenosis, while the rate of SB restenosis was higher without KBT in both true $(36.7 \%$ vs. 
Table 7. Univariate and multivariate analysis of the factors which had an effect on the major adverse cardiac events (MACE).

\begin{tabular}{|c|c|c|c|c|c|c|c|c|}
\hline & \multicolumn{4}{|c|}{ Univariate analysis } & \multicolumn{4}{|c|}{ Multivariate analysis } \\
\hline & \multirow{2}{*}{ Odds ratio } & \multicolumn{2}{|c|}{ 95\% confidence interval } & \multirow{2}{*}{$p$-value } & \multirow{2}{*}{ Odds ratio } & \multicolumn{2}{|c|}{ 95\% confidence interval } & \multirow{2}{*}{$p$-value } \\
\hline & & Lower & Upper & & & Lower & Upper & \\
\hline Kissing balloon & 0.66 & 0.25 & 1.77 & 0.412 & 0.81 & 0.25 & 2.63 & 0.721 \\
\hline Age & 1.01 & 0.96 & 1.07 & 0.641 & 1.00 & 0.94 & 1.07 & 0.984 \\
\hline Hypertension & 0.88 & 0.28 & 2.82 & 0.832 & 0.86 & 0.23 & 3.24 & 0.826 \\
\hline Diabetes mellitus & 1.10 & 0.41 & 2.94 & 0.855 & 1.04 & 0.33 & 3.28 & 0.940 \\
\hline Dyslipidaemia & 0.73 & 0.26 & 2.05 & 0.549 & 0.66 & 0.20 & 2.22 & 0.507 \\
\hline Smoking history & 0.63 & 0.23 & 1.69 & 0.356 & 0.56 & 0.18 & 1.78 & 0.326 \\
\hline ACS/UAP & 1.34 & 0.36 & 4.91 & 0.661 & 1.43 & 0.33 & 6.31 & 0.634 \\
\hline 3-vessel disease & 2.35 & 0.78 & 7.07 & 0.129 & 2.51 & 0.72 & 8.72 & 0.146 \\
\hline True bifurcation lesion & 1.04 & 0.39 & 2.80 & 0.933 & 1.01 & 0.29 & 3.43 & 0.993 \\
\hline Bifurcation angle $>70^{\circ}$ & 0.75 & 0.24 & 2.39 & 0.631 & 0.75 & 0.20 & 2.86 & 0.673 \\
\hline Stent size & 0.75 & 0.17 & 3.38 & 0.713 & 3.12 & 0.40 & 24.22 & 0.276 \\
\hline Stent length & 1.00 & 0.91 & 1.09 & 0.921 & 0.97 & 0.88 & 1.08 & 0.634 \\
\hline Inflation pressure & 0.98 & 0.82 & 1.17 & 0.794 & 0.98 & 0.80 & 1.19 & 0.809 \\
\hline Pre proximal MV MLD & 0.48 & 0.20 & 1.17 & 0.106 & 0.10 & 0.00 & 2.13 & 0.141 \\
\hline Pre proximal MV \%DS & 1.01 & 0.99 & 1.03 & 0.425 & 0.96 & 0.89 & 1.04 & 0.341 \\
\hline Pre distal MV MLD & 0.31 & 0.09 & 1.10 & 0.070 & 0.11 & 0.00 & 3.96 & 0.229 \\
\hline Pre distal MV \%DS & 1.01 & 0.99 & 1.04 & 0.336 & 0.98 & 0.90 & 1.06 & 0.604 \\
\hline Pre SB MLD & 0.74 & 0.26 & 2.10 & 0.566 & 0.79 & 0.05 & 13.22 & 0.869 \\
\hline Pre SB \%DS & 1.00 & 0.97 & 1.02 & 0.778 & 0.99 & 0.93 & 1.05 & 0.634 \\
\hline
\end{tabular}

Table 8. Univariate and multivariate analysis of the factors which had an effect on side branch restenosis.

\begin{tabular}{|c|c|c|c|c|c|c|c|c|}
\hline & \multicolumn{4}{|c|}{ Univariate analysis } & \multicolumn{4}{|c|}{ Multivariate analysis } \\
\hline & \multirow{2}{*}{ Odds ratio } & \multicolumn{2}{|c|}{$95 \%$ confidence interval } & \multirow{2}{*}{$p$-value } & \multirow{2}{*}{ Odds ratio } & \multicolumn{2}{|c|}{$95 \%$ confidence interval } & \multirow{2}{*}{$p$-value } \\
\hline & & Lower & Upper & & & Lower & Upper & \\
\hline Kissing balloon & 0.32 & 0.16 & 0.62 & 0.001 & 0.28 & 0.12 & 0.62 & 0.002 \\
\hline Age & 1.00 & 0.96 & 1.03 & 0.792 & 0.99 & 0.95 & 1.03 & 0.504 \\
\hline Hypertension & 1.70 & 0.72 & 4.05 & 0.228 & 1.16 & 0.43 & 3.14 & 0.769 \\
\hline Diabetes mellitus & 2.07 & 1.09 & 3.94 & 0.027 & 1.40 & 0.65 & 3.04 & 0.391 \\
\hline Dyslipidaemia & 1.08 & 0.53 & 2.18 & 0.835 & 1.16 & 0.49 & 2.73 & 0.734 \\
\hline Smoking history & 1.22 & 0.64 & 2.33 & 0.550 & 1.48 & 0.68 & 3.21 & 0.318 \\
\hline ACS/UAP & 0.36 & 0.11 & 1.24 & 0.106 & 0.29 & 0.06 & 1.36 & 0.117 \\
\hline 3-vessel disease & 3.30 & 1.58 & 6.90 & 0.002 & 3.23 & 1.33 & 7.86 & 0.010 \\
\hline True bifurcation lesion & 2.03 & 1.05 & 3.94 & 0.036 & 1.89 & 0.81 & 4.42 & 0.142 \\
\hline Bifurcation angle $>70^{\circ}$ & 0.83 & 0.40 & 1.70 & 0.604 & 0.96 & 0.41 & 2.23 & 0.916 \\
\hline Stent size & 0.35 & 0.13 & 0.97 & 0.043 & 0.37 & 0.09 & 1.54 & 0.172 \\
\hline Stent length & 0.99 & 0.93 & 1.05 & 0.731 & 0.98 & 0.92 & 1.06 & 0.655 \\
\hline Inflation pressure & 0.98 & 0.87 & 1.09 & 0.664 & 0.98 & 0.85 & 1.12 & 0.727 \\
\hline Pre proximal MV MLD & 1.27 & 0.80 & 2.01 & 0.314 & 1.97 & 0.36 & 10.84 & 0.436 \\
\hline Pre proximal MV \%DS & 0.99 & 0.98 & 1.00 & 0.151 & 0.99 & 0.95 & 1.05 & 0.828 \\
\hline Pre distal MV MLD & 1.02 & 0.56 & 1.84 & 0.954 & 1.15 & 0.14 & 9.45 & 0.898 \\
\hline Pre distal MV \%DS & 0.99 & 0.98 & 1.01 & 0.445 & 0.98 & 0.93 & 1.04 & 0.460 \\
\hline Pre SB MLD & 0.32 & 0.15 & 0.68 & 0.003 & 0.34 & 0.05 & 2.26 & 0.265 \\
\hline Pre SB \%DS & 1.02 & 1.00 & 1.04 & 0.014 & 1.01 & 0.97 & 1.05 & 0.652 \\
\hline
\end{tabular}


$15.5 \%, \mathrm{p}=0.01)$ and non-true bifurcation lesions ( $21.0 \%$ vs. $6.6 \%$, $\mathrm{p}=0.03$ ) (Figure 5). SB restenosis was more frequent in true bifurcation lesions than in non-true lesions regardless of KBT use.

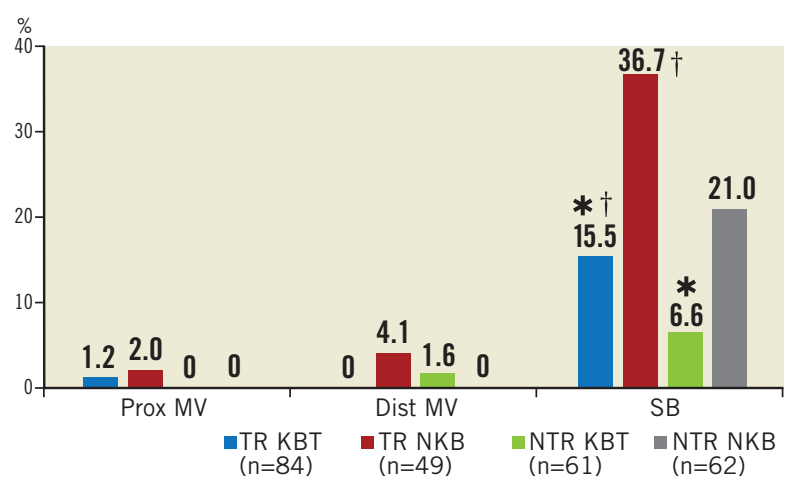

Figure 5. Sub-analysis of binary restenosis at nine-month follow-up in true and non-true bifurcations. KBT: KBT group; NKB: non-KBT group; NTR: non-true bifurcation lesion; TR: true bifurcation lesion; $*$ KBT vs. Non-KBT group in the same subgroup, $p<0.05$; $\uparrow$ true vs. non-true bifurcation lesion, $p<0.05$

\section{Discussion}

This analysis, based on a prospective multicentre registry, investigated the efficacy of KBT in the provisional stenting, under IVUS guidance, of bifurcation lesions not involving the left main coronary artery. Our major findings were that: 1) KBT induced more luminal enlargement with less symmetry in the proximal $\mathrm{MV}$, and larger luminal diameter was maintained up to nine-month followup; 2) KBT brought more SB dissection and required SB stenting in the acute phase, but MACE up to nine-month follow-up did not differ between groups; 3) the angiographic restenosis rate in the SB was greater in non-KBT-treated than KBT-treated lesions; 4) the beneficial effect of KBT on SB restenosis was confirmed in multivariate analysis, but any effect on MACE was not shown; and 5) in both true and non-true bifurcation lesions, KBT produced similar MACE and reduced SB restenosis compared to nonKBT treatment.

\section{LUMINAL ENLARGEMENT OF PROXIMAL MV WITH ASYMMETRIC STENT EXPANSION}

The KBT group had a greater percentage lumen volume gain of proximal to distal MV compared to the non-KBT group $(31.5 \%$ vs. $15.1 \%$ ), consistent with other studies ${ }^{14,15}$. Larger MLD in the proximal MV was maintained up to nine-month follow-up $(2.73 \pm 0.43 \mathrm{~mm}$ vs. $2.63 \pm 0.39 \mathrm{~mm})$. Although the relatively larger proximal MV plaque burden in the non-KBT group (\% plaque area $41.9 \pm 11.4 \%$ vs. $44.4 \pm 8.7 \%, \mathrm{p}=0.06)$ may be attributable in part to less expansion, KBT provided a larger lumen in the proximal MV.

However, KBT led to asymmetric stent expansion in the proximal MV with a lower eccentricity index (0.80-0.82), consistent with previous studies ${ }^{14,15}$. Asymmetric stent expansion raises the following concerns: (1) elliptical stent deformation ${ }^{14-19}$ may lead to destruction of DES strut and polymer alignments ${ }^{15-19}$ and drug concentration inhomogeneity ${ }^{20}$; (2) neointimal hyperplasia is likely after bare metal stent deployment ${ }^{21}$, and uneven neointimal growth is promoted after SES deployment ${ }^{22}$; and (3) a low shear stress area, which may promote neoatherosclerosis, remains in the overdilated area ${ }^{17}$. In the present study, MACE and late loss in the proximal or distal MV did not differ significantly between the KBT and the non-KBT groups. In a clinical study using optical coherence tomography, KBT and non-KBT after DES deployment were associated with similar neointimal hyperplasia rates in the proximal MV at six to 12-month follow-up ${ }^{14}$. Hence, an advantage of KBT is that luminal enlargement in the proximal MV is maintained at midterm follow-up, even when the proximal stent lumen is deformed to an elliptical shape.

\section{CLINICAL OUTCOME}

In the acute phase, KBT was associated with more SB dissection with flow limitation $(10.5 \%$ vs. $1.5 \%)$ and required more stenting $(5.6 \%$ vs. $0.7 \%)$ than non-KBT. Greater SB stenosis in the KBT group $(38.5 \pm 20.5 \%$ vs. $33.9 \pm 18.9 \%)$ may be attributable to a higher prevalence of these events. In terms of hard endpoints, the frequencies of no/slow-flow phenomenon, SB occlusion, and MI were similar. The IVUS survey might have contributed to minimising these events due to accurate assessment of the dissection and optimal SB stent size selection. Functional assessment of jailed SB after MV stenting by fractional flow reserve (FFR) is also effective for reduction of SB treatment; however, a recent randomised trial comparing angiography and FFR guidance for SB treatment demonstrated that no significant difference was observed in MACE, target vessel revascularisation or MI at oneyear follow-up despite an increase of KBT and SB stenting in the angiography-guided treatment ${ }^{23}$. The optimisation of SB treatment by IVUS may be necessary even in FFR-guided SB treatment.

At nine-month follow-up, MACE rates were $6.3 \%$ and $9.1 \%$ in the KBT and non-KBT groups, respectively. MI, cardiac death, and stent thrombosis frequencies were similarly low in both groups, and TLR rates were $5.5 \%$ and $8.1 \%$, respectively. The efficacy of KBT for TLR has been controversial in previous studies ${ }^{6-8,14}$, with widely varying TLR rates. However, the efficacy of final KBT for the reduction of MI and stent thrombosis in two-stent deployment was revealed in the 36-month follow-up results of the Nordic Stent Technique Study ${ }^{24}$. Although this study included more elderly, hypertensive, and diabetic patients and relatively smaller-vessel lesions, the post-PCI MLD of the proximal MV was larger in the present study (KBT $2.91 \pm 0.42 \mathrm{~mm}$ and non-KBT $2.82 \pm 0.37 \mathrm{~mm}$ vs. $2.70 \pm 0.44 \mathrm{~mm}$ and $2.64 \pm 0.45 \mathrm{~mm}$ in Nordic Baltic III) ${ }^{6}$.

Aggressive dilation to achieve an adequate stent area or complete stent apposition based on IVUS success criteria resulted in the high rate of their accomplishment in this study, which could lead to an increased risk of overexpansion and edge dissection requiring TLR. Only one SB lesion was reintervened in each group, and the majority of TLRs were performed in the MV. TLR tended to occur in the stent edge in the KBT group (six out of eight cases of whole TLR), and in-stent in the non-KBT group (seven out of 
nine cases). Elliptical stent deformation and high strain stress during KBT increase the risks of dissection of the proximal stent edge and low shear stress area in the overdilated area ${ }^{17-19}$. Slipping or inappropriate location of the MV balloon during KBT also carries a risk of distal stent edge dissection. Decreased in-stent TLR in the KBT group is thought to arise from the large acquired MV lumen and improvement of DES strut apposition ${ }^{14}$.

In the multivariate analysis (Table 7), the KBT did not have any significant effect on MACE, which was consistent with the Nordic Baltic III trial ${ }^{6}$, but not with the COBIS registry which demonstrated its adverse effect ${ }^{8}$. Since diabetes mellitus was also not associated with MACE, its predominance in the non-KBT group did not influence these clinical outcomes.

\section{ANGIOGRAPHIC RESTENOSIS}

Angiographic restenosis in each branch was assessed at ninemonth follow-up. Binary restenosis in the proximal and distal MV were acceptably low in both groups, while SB restenosis was frequent in the non-KBT group $(9.7 \%$ vs. $27.0 \%)$. In the KBT group, the percentage diameter stenosis of the SB decreased post PCI and dropped further in the follow-up period (Figure 4). KBT increased EEM and lumen CSAs in the SB, decreased plaque area percentage at the MLA site, and expanded EEM and lumen volume regardless of increased plaque volume in the bifurcated area of the SB (Table 3), suggesting that significant vessel stretch caused by KBT overcame the plaque shift from the MV and resulted in persistent luminal enlargement. By contrast, \%DS in the SB increased after PCI, which was maintained in the followup period in the non-KBT group (Figure 4). In the multivariate analysis with various confounding factors (Table 8), the performance of KBT was detected as a robust factor which reduced $\mathrm{SB}$ restenosis. In the sub-analysis comparing true and non-true bifurcation lesions, SB restenosis was less frequent in the KBT group in both true (16\% vs. $37 \%$ ) and non-true bifurcation lesions (7\% vs. 21\%). The Nordic Baltic III study also demonstrated a favourable effect of KBT in reducing $\mathrm{SB}$ restenosis, but only in true bifurcation lesions ${ }^{6}$. However, SB vessel references in this study were comparatively smaller, and post-PCI lumen enlargement in the distal MV was greater in the present study ${ }^{6}$; thus, the SB ostium was narrowed more due to carina shift in the present study. Although there is concern regarding SB ostial stenosis progression in the non-KBT group due to coronary flow turbulence at the $\mathrm{SB}$ ostium $^{25}$ and intimalisation on jailed struts, this phenomenon was not observed over the nine-month follow-up period in the present study.

\section{STUDY LIMITATIONS}

This study was based on a prospective registry of patients treated according to their physician's preference. Consequently, the groups were not randomised and were imperfectly matched with respect to clinical and lesion characteristics, so biases in stent selection cannot be ruled out. The higher frequency of diabetic patients and tendency for greater plaque burden in the proximal
MV might have impacted on the higher TLR frequency in the non-KBT group compared to other randomised bifurcation studies. More frequent binary restenosis in the SB in the non-KBT group may also be attributable to the tendency for smaller reference vessels. The jailed SB was assessed only by quantitative angiographic analysis, not by FFR; hence, stenosis was not functionally assessed. Furthermore, the SB lesions treated without KBT could not be evaluated by IVUS after PCI, and our observations are not applicable to bifurcation lesions of the left main coronary artery. Finally, a longer follow-up is needed to compare the rates of adverse clinical events between KBT and non-KBTtreated groups and to clarify the fate of jailed struts.

\section{Impact on daily practice}

The efficacy of the kissing balloon technique (KBT) after intravascular ultrasound (IVUS)-guided provisional stenting is confirmed in the present study. KBT induces greater luminal enlargements in the proximal main vessel (MV) and side branch $(\mathrm{SB})$, which are maintained at the midterm follow-up period regardless of higher detection of SB dissection requiring stenting. Although the rates of major adverse cardiac events (MACE) at the follow-up are similar to non-KBT treatment, KBT reduces SB restenosis significantly. Accurate assessment of the bifurcated vessel in terms of size, plaque and intima by IVUS leads to optimal KBT treatment in the SB without increasing MV events, which are related to MACE.

\section{Appendix LIST OF PARTICIPATING CENTRES AND INVESTIGATORS}

Masaya Arikawa, Tetsuya Kira, Oita Medical Center; Yoritaka Otsuka, Fukuoka Wajiro Hospital; Masaki Tanabe, The Second Okamoto Hospital; Yoshinobu Murasato, Kyushu Medical Center and New Yukuhashi Hospital; Masataka Horiuchi, Yasunori Suematsu, Tomohiko Yamamoto, Taichi Okonogi, New Yukuhashi Hospital; Yoshihisa Kinoshita, Takahiko Suzuki, Toyohashi Heart Center; Masahiro Yamawaki, Toshiya Muramatsu, Reiko Tsukahara, Norihiro Kobayashi, Saiseikai Yokohama City Eastern Hospital; Toshiro Shinke, Hirotoshi Hariki, Kobe University; Yoshihiro Takeda, Rinku General Medical Center; Shin-ichiro Yamada, Himeji Cardiovascular Center; Kenichi Fujii, Hyogo Medical University; Yoshihisa Shimada, Naoto Kino, Daisuke Tonomura, Shiroyama Hospital; Takehiro Yamashita, Masato Nagashima, Cardiovascular Center Hokkaido Ono Hospital; Kazuhiko Yumoto, Yokohama Rosai Hospital.

Core laboratory: Yasuyoshi Suzuki, Mitsuyasu Terashima, Cardiovascular Imaging Center; Hiromasa Otake, Hachidai Takahashi, Hiroto Kinutani, Daisuke Terashita, Kobe University.

Statistical Adviser: Yoshihisa Fujino, Department of Preventive Medicine and Community Health, University of Occupational Environmental Health.

Safety Committee: Yoshinori Takajyo, Takajyo Clinic; Masato Tsutsui, Ryukyu University. 


\section{Funding}

J-REVERSE was supported by unrestricted research grants from Abbott Vascular, Cordis Corporation, OrbusNeich, and Kaneka Corporation.

\section{Conflict of interest statement}

The authors have no conflicts of interest to declare.

\section{References}

1. Hildick-Smith D, Lassen JF, Albiero R, Lefèvre T, Darremont O, Pan M, Ferenc M, Stankovic G, Louvard Y; European Bifurcation Club. Consensus from the 5th European Bifurcation Club meeting. EuroIntervention. 2010;6:34-8.

2. Steigen TK, Maeng M, Wiseth R, Erglis A, Kumsars I, Narbute I, Gunnes P, Mannsverk J, Meyerdierks O, Rotevatn S, Niemelä M, Kervinen K, Jensen JS, Galløe A, Nikus K, Vikman S, Ravkilde J, James S, Aarøe J, Ylitalo A, Helqvist S, Sjögren I, Thayssen P, Virtanen K, Puhakka M, Airaksinen J, Lassen JF, Thuesen L; Nordic PCI Study Group. Randomized study on simple versus complex stenting of coronary artery bifurcation lesions: the Nordic bifurcation study. Circulation. 2006;114:1955-61.

3. Hildick-Smith D, de Belder AJ, Cooter N, Curzen NP, Clayton TC, Oldroyd KG, Bennett L, Holmberg S, Cotton JM, Glennon PE, Thomas MR, Maccarthy PA, Baumbach A, Mulvihill NT, Henderson RA, Redwood SR, Starkey IR, Stables RH. Randomized trial of simple versus complex drug-eluting stenting for bifurcation lesions: the British Bifurcation Coronary Study: old, new, and evolving strategies. Circulation. 2010;121:1235-43.

4. Zimarino M, Corazzini A, Ricci F, Di Nicola M, De Caterina R. Late thrombosis after double versus single drug-eluting stent in the treatment of coronary bifurcations: a meta-analysis of randomized and observational studies. JACC Cardiovasc Interv. 2013;6:687-95.

5. Sgueglia GA, Chevalier B. Kissing balloon inflation in percutaneous coronary interventions. JACC Cardiovasc Interv. 2012;5:803-11.

6. Niemelä M, Kervinen K, Erglis A, Holm NR, Maeng M, Christiansen EH, Kumsars I, Jegere S, Dombrovskis A, Gunnes P, Stavnes S, Steigen TK, Trovik T, Eskola M, Vikman S, Romppanen H, Mäkikallio T, Hansen KN, Thayssen P, Aberge L, Jensen LO, Hervold A, Airaksinen J, Pietilä M, Frobert O, Kellerth T, Ravkilde J, Aarøe J, Jensen JS, Helqvist S, Sjögren I, James S, Miettinen H, Lassen JF, Thuesen L; Nordic-Baltic PCI Study Group. Randomized comparison of final kissing balloon dilatation versus no final kissing balloon dilatation in patients with coronary bifurcation lesions treated with main vessel stenting: the Nordic-Baltic Bifurcation Study III. Circulation. 2011;123:79-86.

7. Korn HV, Yu J, Ohlow MA, Huegl B, Schulte W, Wagner A, Wassmer G, Gruene S, Petek O, Lauer B. Interventional therapy of bifurcation lesions: a TIMI flow-guided concept to treat side branches in bifurcation lesions--a prospective randomized clinical study (Thueringer bifurcation study, THUEBIS study as pilot trial). Circ Cardiovasc Interv. 2009;2:535-42.
8. Gwon HC, Hahn JY, Koo BK, Song YB, Choi SH, Choi JH, Lee SH, Jeong MH, Kim HS, Seong IW, Yang JY, Rha SW, Jang Y, Yoon JH, Tahk SJ, Seung KB, Park SJ. Final kissing ballooning and long-term clinical outcomes in coronary bifurcation lesions treated with 1-stent technique: results from the COBIS registry. Heart. 2012;98:225-31.

9. Park SJ, Kim YH, Park DW, Lee SW, Kim WJ, Suh J, Yun SC, Lee CW, Hong MK, Lee JH, Park SW; MAIN-COMPARE Investigators. Impact of intravascular ultrasound guidance on longterm death in stenting for unprotected left main coronary artery stenosis. Circ Cardiovasc Interv. 2009;2:167-77.

10. Patel Y, Depta JP, Novak E, Yeung M, Lavine K, Banerjee S, Lin CH, Zajarias A, Kurz HI, Lasala JM, Bach RG, Singh J. Longterm outcomes with use of intravascular ultrasound for the treatment of coronary bifurcation lesions. Am J Cardiol. 2012;109:960-5.

11. Zhang Y, Farooq V, Garcia-Garcia HM, Bourantas CV, Tian N, Dong S, Li M, Yang S, Serruys PW, Chen SL. Comparison of intravascular ultrasound versus angiography-guided drug-eluting stent implantation: a meta-analysis of one randomised trial and ten observational studies involving 19,619 patients. EuroIntervention. 2012;8:855-65.

12. Moussa I, Moses J, Di Mario C, Albiero R, De Gregorio J, Adamian M, Di Francesco L, Colombo A. Does the specific intravascular ultrasound criterion used to optimize stent expansion have an impact on the probability of stent restenosis? Am J Cardiol. 1999;83:1012-7.

13. Kanda Y. Investigation of the freely-available easy-to-use software "EZR" for medical statistics. Bone Marrow Transplant. 2013;48:452-8

14. Hariki H, Shinke T, Otake H, Shite J, Nakagawa M, Inoue T, Osue T, Iwasaki M, Taniguchi Y, Nishio R, Hiranuma N, Kinutani H, Konishi A, Hirata K. Potential benefit of final-kissing inflation after single stenting for the treatment of bifurcation lesions: insight from OCT observations. Circ J. 2013;77:1193-201.

15. Rahman S, Leesar T, Cilingiroglu M, Effat M, Arif I, Helmy T, Leesar MA. Impact of kissing balloon inflation on the main vessel stent volume, area, and symmetry after side-branch dilation in patients with coronary bifurcation lesions: a serial volumetric intravascular ultrasound study. JACC Cardiovasc Interv. 2013;6: 923-31.

16. Guérin P, Pilet P, Finet G, Gouëffic Y, N'Guyen JM, Crochet D, Tijou I, Pacaud P, Loirand G. Drug-eluting stents in bifurcations: bench study of strut deformation and coating lesions. Circ Cardiovasc Interv. 2010;3:120-6.

17. Murasato Y, Iwasaki K, Yamamoto T, Yagi T, Hikichi Y, Suematsu Y, Yamamoto T. Optimal kissing balloon inflation after single-stent deployment in a coronary bifurcation model. EuroIntervention. 2014;10:934-41.

18. Mortier P, De Beule M, Dubini G, Hikichi Y, Murasato Y, Ormiston JA. Coronary bifurcation stenting: insights from in vitro and virtual bench testing. EuroIntervention. 2010;6:J53-60.

19. Foin N, Torii R, Mortier P, De Beule M, Viceconte N, Chan PH, Davies JE, Xu XY, Krams R, Di Mario C. Kissing 
balloon or sequential dilation of the side branch and main vessel for provisional stenting of bifurcations: lessons from micro-computed tomography and computational simulations. JACC Cardiovasc Interv. 2012;5:47-56.

20. Hwang CW, Wu D, Edelman ER. Physiological transport forces govern drug distribution for stent-based delivery. Circulation. 2001;104:600-5.

21. Kaneda H, Ako J, Honda Y, Terashima M, Morino Y, Yock PG, Popma JJ, Leon MB, Moses JW, Fitzgerald PJ. Impact of asymmetric stent expansion on neointimal hyperplasia following sirolimus-eluting stent implantation. Am J Cardiol. 2005;96: 1404-7.

22. Otake H, Shite J, Ako J, Shinke T, Tanino Y, Ogasawara D, Sawada T, Miyoshi N, Kato H, Koo BK, Honda Y, Fitzgerald PJ, Hirata K. Local determinants of thrombus formation following sirolimus-eluting stent implantation assessed by optical coherence tomography. JACC Cardiovasc Interv. 2009;2:459-66.

23. Chen SL, Ye F, Zhang JJ, Xu T, Tian NL, Liu ZZ, Lin S, Shan SJ, Ge Z, You W, Liu YQ, Qian XS, Li F, Yang S, Kwan TW,
$\mathrm{Xu} \mathrm{B}$, Stone GW. Randomized Comparison of FFR-Guided and Angiography-Guided Provisional Stenting of True Coronary Bifurcation Lesions: The DKCRUSH-VI Trial (Double Kissing Crush Versus Provisional Stenting Technique for Treatment of Coronary Bifurcation Lesions VI). JACC Cardiovasc Interv. 2015;8:536-46.

24. Kervinen K, Niemelä M, Romppanen H, Erglis A, Kumsars I, Maeng M, Holm NR, Lassen JF, Gunnes P, Stavnes S, Jensen JS, Galløe A, Narbute I, Sondore D, Christiansen EH, Ravkilde J, Steigen TK, Mannsverk J, Thayssen P, Hansen KN, Helqvist S, Vikman S, Wiseth R, Aarøe J, Jokelainen J, Thuesen L; Nordic PCI Study Group. Clinical outcome after crush versus culotte stenting of coronary artery bifurcation lesions: the Nordic Stent Technique Study 36-month follow-up results. JACC Cardiovasc Interv. 2013;6:1160-5.

25. Katritsis DG, Theodorakakos A, Pantos I, Gavaises M, Karcanias N, Efstathopoulos EP. Flow patterns at stented coronary bifurcations: computational fluid dynamics analysis. Circ Cardiovasc Interv. 2012;5:530-9. 